\title{
Combination of the sodium-glucose
}

\section{cotransporter-2 inhibitor empagliflozin with orlistat or sibutramine further improves the body-weight reduction and glucose homeostasis of obese rats fed a cafeteria diet}

This article was published in the following Dove Press journal:

Diabetes, Metabolic Syndrome and Obesity: Targets and Therapy I July 2014

Number of times this article has been viewed

\author{
Steven P Vickers' \\ Sharon C Cheetham' \\ Katie R Headland' \\ Keith Dickinson' \\ Rolf Grempler ${ }^{2}$ \\ Eric Mayoux ${ }^{2}$ \\ Michael Mark ${ }^{2}$ \\ Thomas Klein ${ }^{2}$ \\ 'RenaSci, BioCity Nottingham, \\ Nottingham, UK; ${ }^{2}$ Boehringer \\ Ingelheim Pharma, Biberach an der \\ Riss, Germany
}

\begin{abstract}
The present study assessed the potential of the sodium glucose-linked transporter (SGLT)-2 inhibitor empagliflozin to decrease body weight when administered alone or in combination with the clinically effective weight-loss agents orlistat and sibutramine in obese rats fed a cafeteria diet. Female Wistar rats were exposed to a cafeteria diet to induce obesity. Empagliflozin was dosed once daily $(10,30$, and $60 \mathrm{mg} / \mathrm{kg}$ ) for 28 days. Combination studies were subsequently performed using a submaximal empagliflozin dose $(10 \mathrm{mg} / \mathrm{kg})$ with either sibutramine or orlistat. Body weight, food, and water intake were recorded daily. The effect of drug treatment on glucose tolerance, relevant plasma parameters, and carcass composition was determined. Empagliflozin dose-dependently reduced body weight, plasma leptin, and body fat though increased urinary glucose excretion. The combination of empagliflozin and orlistat significantly reduced body weight compared to animals treated with either drug alone, and significantly improved glucose tolerance, plasma insulin, and leptin compared to vehicle-treated controls. The effect of sibutramine to improve glycemic control in an oral glucose-tolerance test was also significantly increased, with empagliflozin and combination treatment leading to a reduction in carcass fat greater than that observed with either drug alone. These data demonstrate that empagliflozin reduces body weight in cafeteria-fed obese rats. In combination studies, empagliflozin further improved the body-weight or body-fat loss of animals in comparison to orlistat or sibutramine alone. Such studies may indicate improved strategies for the treatment of obese patients with prediabetes or type 2 diabetes.
\end{abstract}

Keywords: SGLT2, empagliflozin, sibutramine, obesity, rat, combination

\section{Introduction}

The World Health Organization estimates that 400 million adults are obese and 1.6 billion are overweight worldwide. ${ }^{1}$ Importantly, the obesity epidemic is no longer restricted to Western cultures, but is becoming a global burden, with such countries as Mexico, Brazil, and the People's Republic of China currently most affected. ${ }^{2}$ In the absence of suitable intervention, the global epidemic of obesity is predicted to become a leading cause of morbidity and mortality, driven by an increase in related life-threatening disorders, including dyslipidemia, hypertension, cancer, and type 2 diabetes. ${ }^{3}$ Specifically, type 2 diabetes mellitus is one of the most common and frequent health consequences of obesity, with more than $80 \%$ of patients with type 2 diabetes being obese or overweight. In addition, a large number of obese patients are likely
Correspondence: Eric Mayoux Boehringer Ingelheim Pharma, Birkendorfer Strasse 65, 88400 Biberach an der Riss, Germany Email eric.mayoux@boehringeringelheim.com 
to exhibit prediabetes, a state characterized by impaired glucose tolerance and insulin resistance, although this will be undiagnosed and therefore untreated.

There are a number of therapeutic interventions for the treatment of obesity, including low-calorie diets, increased physical activity, behavioral therapy, pharmacological intervention, and bariatric surgery. However, these are generally limited in efficacy and/or safety. ${ }^{4}$ Body-weight reduction is often a prerequisite in treating prediabetic and diabetic patients. A drug or a combination of drugs able to exert antiobesity and antidiabetic properties could slow down or prevent the progression from obesity to type 2 diabetes. Not only is obesity a major risk factor for the development of type 2 diabetes, many oral antidiabetic agents are associated with weight gain. ${ }^{5}$ Accordingly, clinically effective antidiabetic drugs that decrease body weight may be of increased utility in the successful treatment of diabetes and obesity. Sodium glucose-linked transporter (SGLT)-2 inhibition may be an advantageous pharmacological approach to such a patient population, since such drugs block the reabsorption of glucose in the proximal tubule of the kidney, ${ }^{6}$ and the resulting augmentation of urinary glucose excretion (UGE) has been associated with weight loss in the clinic in addition to the antidiabetic effect. ${ }^{7,8}$ Moreover the efficacy of this emerging new drug class is insulin-independent and associated with a reduced risk of hypoglycemia, ${ }^{9,10}$ a characteristic of particular relevance if the compound were to be used in prediabetic patients who may have only moderate hyperglycemia.

Empagliflozin (BI-10773) is a novel, potent, and selective SGLT2 inhibitor that exhibits efficacy in animal models of diabetes and is currently in development for the treatment of type 2 diabetes. ${ }^{11,12}$ The present study determined the effect of empagliflozin on body weight, carcass composition, levels of relevant plasma markers and UGE in an animal model of dietary-induced obesity (DIO) with excellent predictive validity. ${ }^{13-15}$ In light of both the reported effect of SGLT2 inhibitors to cause weight loss in the clinic and the practice of polypharmacy for the treatment of type 2 diabetes, ${ }^{16,17}$ the present study also investigated the effect of coadministration of empagliflozin with clinically effective drugs for the treatment of obesity, such as the lipase inhibitor orlistat and the serotonin- and noradrenaline-reuptake inhibitor (SNRI) sibutramine in the same animal model. These data may offer insight as to whether such drug combinations (empagliflozin with a drug treatment for obesity) have utility for further evaluation in clinical practice.

\section{Materials and methods}

\section{Animals}

Female Wistar rats (weight range 250-300 g upon arrival) were obtained from Charles River (Margate, UK) and housed in pairs or threes at a temperature of $21^{\circ} \mathrm{C} \pm 4^{\circ} \mathrm{C}$ and $55 \% \pm 20 \%$ humidity. Animals were maintained on a reversephase light-dark cycle (lights off for 8 hours from $9.30 \mathrm{am}$ to $5.30 \mathrm{pm}$ ), during which time the room was illuminated by red light. Animals had free access to a powdered high-fat diet (VRF1 plus 20\% lard; Special Diet Services, Witham, UK), ground chocolate (Dairy Milk; Cadbury, Birmingham UK), ground peanuts (Big D; Trigon Snacks, Liverpool, UK), and tap water at all times unless specified otherwise. Animals were housed on this diet for 15-20 weeks for the induction of obesity prior to the pharmacological study. The work reported in this manuscript was performed in accordance with UK law, as detailed in the Animals (Scientific Procedures) Act 1986.

Two separate studies were conducted (the combination study was performed subsequent to the first study in a separate cohort of animals). Approximately 2 weeks before the start of each study, animals were housed singly in polypropylene cages with wire-grid floors (so food spillage could be determined). Each cage contained an appropriate amount of paper bedding for warmth and environmental enrichment and to provide an area for animals to get off the wire-grid floor.

In each study, animals underwent a baseline period of dosing, where each animal was dosed once daily orally with vehicle by gavage. Toward the end of this baseline phase, animals were allocated by a statistician into treatment groups, balanced in regard to baseline body weight and daily food and water intake. Drug dosing was timed to begin at the onset of the dark phase. Rats, feeding jars and water bottles were weighed (to the nearest $0.1 \mathrm{~g}$ ) every day at the time of drug or vehicle administration.

\section{Methods}

In the initial study, animals were dosed once daily for 28 days with vehicle, empagliflozin $(10,30,60 \mathrm{mg} / \mathrm{kg}$ per os [PO \{by mouth $\}]$ ), or with the positive control sibutramine ( $5 \mathrm{mg} / \mathrm{kg}$ PO). Blood samples (4-hour fasted) were taken from the lateral tail vein during the baseline phase and on days 14 and 28, 4 hours after dosing. On day 21, animals were dosed and immediately placed in metabolism cages for a 7-hour period. Food was not present in the metabolism cages, although animals had free access to water. Urine was collected and assayed for glucose content using hand-held glucose meters (Xceed; Abbott Laboratories, Abbott Park, 
IL, USA). At the end of the study (day 29), carcasses were saved for body-composition analysis.

In the combination studies, sibutramine $(5 \mathrm{mg} / \mathrm{kg} \mathrm{PO})$ was dosed once daily with empagliflozin (10 mg/kg PO). Orlistat was dosed bis in die (BID [twice daily]) $(20 \mathrm{mg} / \mathrm{kg}$ $\mathrm{PO}$ ): once at the start of the dark phase (in combination with empagliflozin) and again 4 hours later to maintain exposure over the dark phase of the light-dark cycle. All dosing was via the oral route by gavage. Blood samples were taken during the baseline phase and on days 16 and 30 after an overnight fast. Shortly after the day 30 blood sampling, animals underwent an oral glucose $(2 \mathrm{~g} / \mathrm{kg})$-tolerance test. Blood samples were taken immediately before and at 10 , 20, 30, 60, and 120 minutes post-glucose administration. At the conclusion of the test, food was returned. On day 34, the experiments concluded, and the carcasses were saved for body-composition analysis.

\section{Drugs}

Empagliflozin was provided by the Department of Medicinal Chemistry, Boehringer Ingelheim Pharma (Biberach an der Riss, Germany). Orlistat and sibutramine $\mathrm{HCl}$ monohydrate were synthesized by Tocris Cookson Ltd (Bristol, UK). All compounds were dosed orally in a vehicle of aqueous $0.5 \%$ Natrosol $^{\mathrm{TM}}$ (Ashland, Covington, KY, USA) weight/volume at a volume of $3 \mathrm{~mL} / \mathrm{kg}$. All doses were corrected for salt where appropriate.

\section{Plasma analysis}

Blood was collected in ethylenediaminetetraacetic acid (EDTA)-coated collection tubes (SARSTEDT Ltd., Leicester, UK), and immediately spun in a cooled centrifuge and the plasma stored frozen. Subsequently, plasma was assayed for content of one or more of the following: glucose, insulin, leptin, adiponectin, glycerol, and triacylglycerol (TAG) content. Blood (collected in an EDTA tube and frozen immediately) was assayed for glycated hemoglobin $\left(\mathrm{HbA}_{1 \mathrm{c}}\right)$.

Commercially available enzyme-linked immunosorbent assay kits and colorimetric kits were used to assay glucose (Thermo Fisher Scientific, Waltham, MA, USA), insulin (Mercodia, Uppsala, Sweden), glycerol and true triglycerides (Sigma-Aldrich, St Louis, MO, USA), nonesterified fatty acid (Wako Pure Chemical Industries, Osaka, Japan), adiponectin (EMD Millipore, Billerica, MA, USA), and leptin (Enzo Life Sciences, Farmingdale, NY, USA). $\mathrm{HbA}_{1 \mathrm{c}}$ was assayed by a direct enzymatic assay (Diazyme Laboratories, Poway, CA, USA).

\section{Body-composition assessment}

Carcass composition (total body fat, protein, and water) was determined using the FoodScan ${ }^{\mathrm{TM}}$ meat analyzer (Foss, Warrington, UK). Carcasses were milled at the temperature of liquid nitrogen and stored at $-20^{\circ} \mathrm{C}$ in sealed containers. This method has been demonstrated to produce results highly comparable $\left(r^{2}=0.95\right)$ to those obtained with the gold-standard chemical analysis method of carcass composition. ${ }^{18}$

\section{Statistical analysis}

Statistical analysis was performed by a statistician. Body weight and food- and water-intake data were assessed by analysis of covariance, with treatment as a factor and baseline data as the covariate. In the case of body-weight analysis, day 1 body weight (ie, the weight immediately before the first drug treatment) was the covariate. In the case of food- and water-intake analysis, the covariate was the average daily intake during the baseline phase of the study.

Plasma data were analyzed by a general linear model, with treatment as a factor. Where appropriate, data underwent a $\log$ transformation prior to analysis. Baseline plasma data and day 1 body weight were included as covariates. $\mathrm{HbA}_{1 \mathrm{c}}$ data were analyzed by a robust regression model using $M$-estimation (Huber weighting, using the default parameter $c=1.345$ ). Baseline $\mathrm{HbA}_{1 \mathrm{c}}$ levels and day 1 body weight were included as covariates. Since no differences were observed between the 2- and 4-week changes in plasma parameters, only the final plasma data for each study are detailed. Area under the curve (AUC) from time 0 to 30 minutes $\left(\mathrm{AUC}_{0-30}\right)$ was calculated by the trapezoidal rule: $1 / 12\left(t_{B 2}+2 t_{10 \text { min }}+2 t_{20 \text { min }}+t_{30 \text { min }}\right)$. Homeostasis-model assessment of insulin resistance (HOMA-IR) values were calculated according to the formula:

$$
\begin{aligned}
\text { HOMA-IR }= & {[\text { fasting insulin }(\mu \mathrm{U} / \mathrm{mL})} \\
& \times \text { fasting glucose }(\mathrm{mmol} / \mathrm{L})] / 22.5 .
\end{aligned}
$$

Carcass composition data were analyzed by robust regression, with treatment as factors. Day 1 body weight was included as a covariate.

Means detailed in figures and tables were adjusted for differences at baseline. UGE data were analyzed as a function of body weight, consistent with much of the preclinical literature. ${ }^{12}$ However, this analysis was not found to differ in regard to statistical significance from an analysis of absolute glucose levels (latter analysis not included). Standard error of the mean was calculated from the residuals of the statistical model. Comparisons between groups were by William's 
test or the multiple $t$-test, as deemed appropriate. A value of $P<0.05$ was regarded as being statistically significant. All statistical analysis was performed using SAS version 9.1.3 (SAS Institute, Cary, NC, USA).

\section{Results}

\section{Effect of empagliflozin in DIO rats}

Empagliflozin (10, 30, and $60 \mathrm{mg} / \mathrm{kg}$ PO) dose-dependently reduced body weight in female DIO rats compared to vehicletreated controls with an $8.2 \%$ weight loss evident compared to controls at the highest dose tested $(P<0.001$, Figure 1$)$. Sibutramine ( $5 \mathrm{mg} / \mathrm{kg}$ PO) reduced body weight by $13.5 \%$ compared to controls $(P<0.001$, Figure 1A). Empagliflozin (10 and $30 \mathrm{mg} / \mathrm{kg}$ PO) did not significantly affect average daily food intake during the study (Figure 1B), with the exception of the highest dose $(60 \mathrm{mg} / \mathrm{kg}$ PO), which moderately reduced average daily food intake $(P<0.01)$ over the entire study duration compared to vehicle-treated controls. As expected, sibutramine significantly decreased average daily food intake during the study ( $P<0.001$, Figure 1B). In contrast to sibutramine, empagliflozin dose-dependently increased water intake over the study duration $(P<0.05$, Figure 1C). Empagliflozin dose-dependently reduced body fat $(P<0.05)$, but did not affect carcass protein or water (Table 1). At the highest dose, $78 \%$ of the total weight loss was attributable to fat loss. Sibutramine also significantly reduced body fat $(P<0.001)$ without affecting carcass protein, although significant reductions in body water $(P<0.05)$

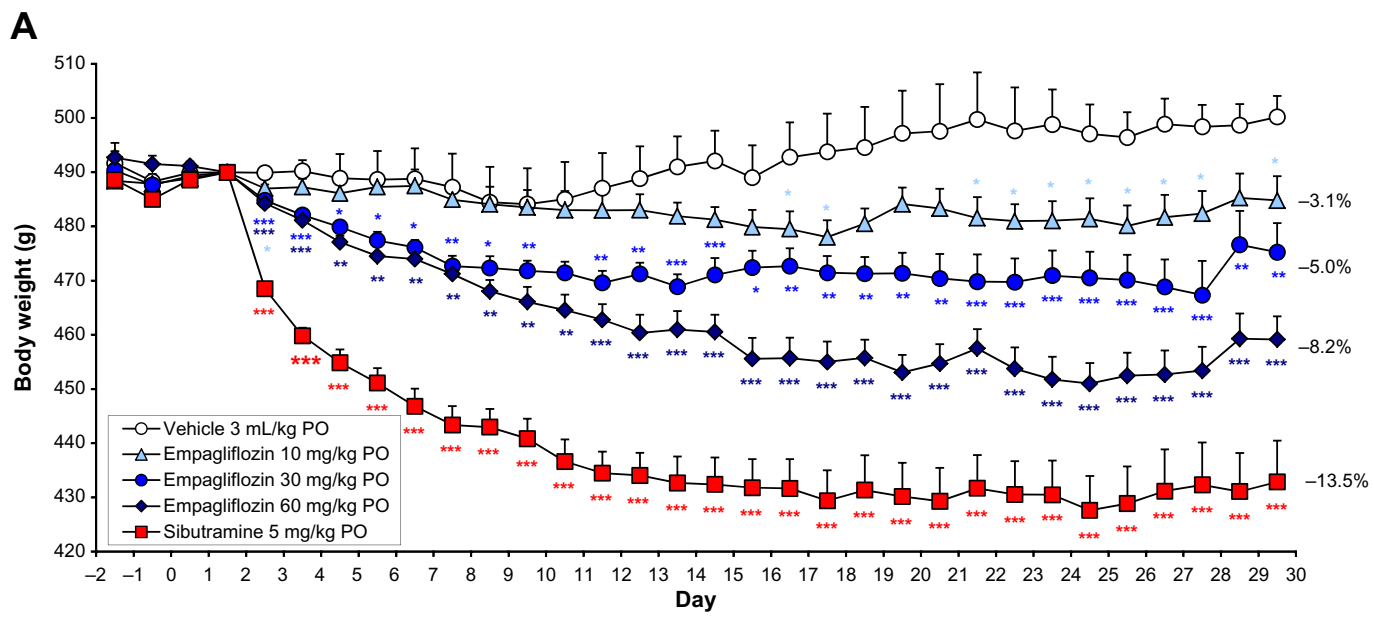

C

B

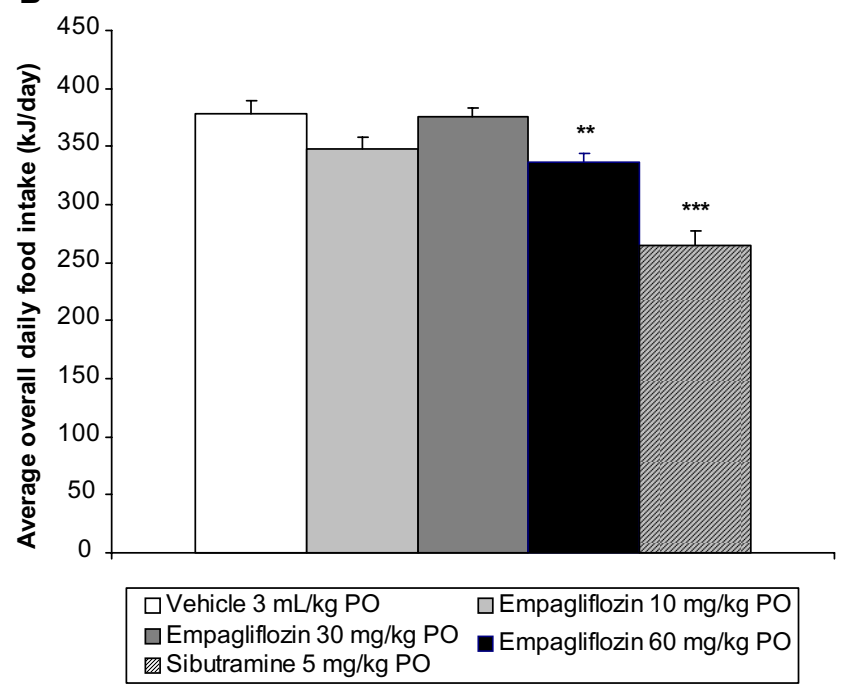

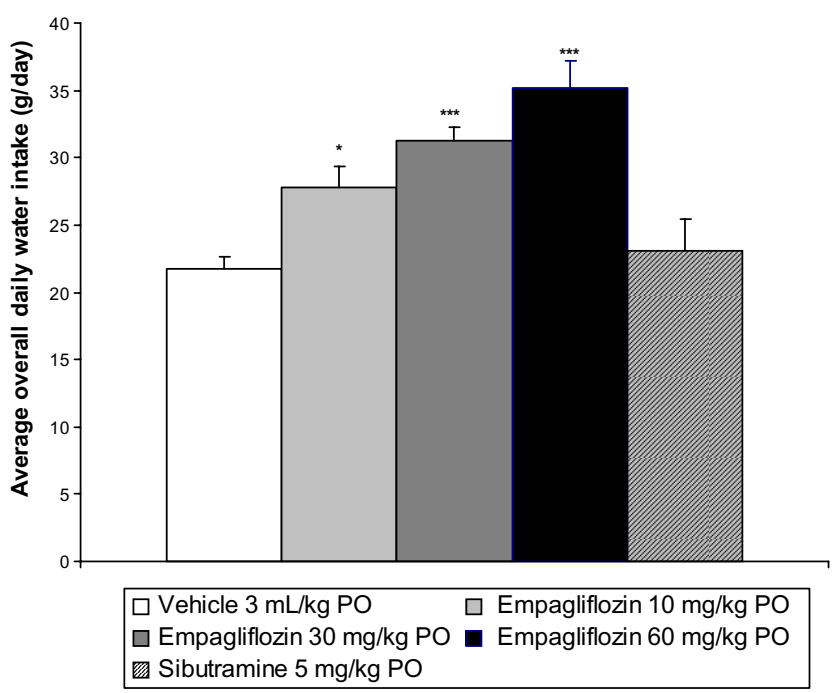

Figure I The effect of empagliflozin on rat body weight $(\mathbf{A})$ average daily food intake $(\mathbf{B})$ and average daily water intake (C).

Notes: $* P<0.05 ; * * P<0.01 ; * * * P<0.001$. Data are means and standard error $(n=8-10)$. Percentage values indicate weight loss relative to vehicle-treated controls on Day 29 . Abbreviation: PO, per os (by mouth). 
Table I Effect of empagliflozin and sibutramine on urinary glucose excretion and body composition of diet-induced obese rats at study conclusion

\begin{tabular}{|c|c|c|c|c|}
\hline & $\begin{array}{l}\text { UGE } \\
(\mathrm{mg} / 200 \mathrm{~g})\end{array}$ & $\begin{array}{l}\text { Water } \\
\text { (g) }\end{array}$ & Fat (g) & $\begin{array}{l}\text { Protein } \\
\text { (g) }\end{array}$ \\
\hline Vehicle & $1.4 \pm 0.1$ & $219.4 \pm 3.6$ & $180.1 \pm 6.3$ & $70.5 \pm 1.5$ \\
\hline $\begin{array}{l}\text { Empagliflozin } \\
\text { (10 mg/kg PO) }\end{array}$ & $\mid 50.1 \pm 32.9 * * *$ & $216.5 \pm 4.3$ & $162.0 \pm 6.1 *$ & $70.2 \pm 1.7$ \\
\hline $\begin{array}{l}\text { Empagliflozin } \\
\text { (30 mg/kg PO) }\end{array}$ & $269.6 \pm 31.7 * * *$ & $213.1 \pm 3.5$ & $158.6 \pm 6.6 *$ & $67.6 \pm 1.4$ \\
\hline $\begin{array}{l}\text { Empagliflozin } \\
(60 \text { mg/kg PO) }\end{array}$ & $367.9 \pm 90.3 * * *$ & $213.7 \pm 3.8$ & $143.8 \pm 3.3 * * *$ & $67.7 \pm 1.8$ \\
\hline $\begin{array}{l}\text { Sibutramine } \\
\text { (5 mg/kg PO) }\end{array}$ & $1.0 \pm 0.2$ & $206.7 \pm 4.9 *$ & $130.6 \pm 8.7 * * *$ & $68.1 \pm 1.6$ \\
\hline
\end{tabular}

Notes: $* P<0.05, * * * P<0.001$ (significant differences from the vehicle-treated group). Data are means and standard error $(n=8-10)$.

Abbreviations: UGE, urinary glucose excretion; PO, per os (by mouth).

were evident. At the dose of sibutramine tested, $67 \%$ of the total weight loss was attributable to fat loss. Empagliflozin, but not sibutramine, dose-dependently increased UGE $(P<0.001)$ on day 21 (Table 1$)$.

Empagliflozin led to statistically significant reductions in plasma glucose $(60 \mathrm{mg} / \mathrm{kg} \mathrm{PO}, P<0.05)$, adiponectin $(60 \mathrm{mg} / \mathrm{kg}$ PO, $P<0.05)$ and leptin $(30$ and $60 \mathrm{mg} / \mathrm{kg}$ PO, $P<0.01$ ) after 28 days of treatment (Table 2), with no significant effect on TAG. Sibutramine had significantly reduced plasma glucose $(P<0.01)$, insulin $(P<0.01)$, adiponectin $(P<0.01)$, TAG $(P<0.01)$, and leptin $(P<0.01)$ by day 28 of the study (Table 2). $\mathrm{HbA}_{1 \mathrm{c}}$ and plasma levels of nonesterified fatty acid were not significantly altered by empagliflozin or sibutramine (Table 2). HOMA-IR was significantly decreased with all doses of empagliflozin, indicating an improvement in insulin sensitivity $(P<0.05$, Table 2$)$. This reduction was even more pronounced with sibutramine $(P<0.001$, Table 2$)$.

\section{Effect of empagliflozin in combination with sibutramine or orlistat}

In the combination study, empagliflozin alone (10 mg/kg PO) tended to reduce body weight over the study duration, although this effect was not statistically significant $(-1.4 \%$ at day 29, Figure 2A). Although daily food intake compared to vehicle-treated controls was not altered, overall daily water intake was significantly elevated $(P<0.05$, data not shown for clarity). The effect of sibutramine to reduce body weight in DIO rats $(P<0.001)$ was moderately increased by cotreatment with empagliflozin, without reaching statistical significance $(P=0.14)$ compared to sibutramine alone (weight loss of $7.0 \%$ with sibutramine alone compared to $9.9 \%$ in combination with empagliflozin, Figure 2A). Orlistat tended to decrease body weight over the study period compared to vehicle-treated controls, although this effect did not reach statistical significance ( $P=0.20$, Figure $2 \mathrm{~A})$. Empagliflozin significantly augmented the effect of orlistat on body weight (from $2.4 \%$ to $6.7 \%$ by day $29, P<0.05$, Figure $2 A$ ).

While sibutramine significantly reduced average daily food intake over the study duration $(P<0.05)$, orlistat significantly increased food intake compared to vehicle-treated controls $(P<0.001$, Figure 2B). Combination dosing with empagliflozin did not significantly alter the effect on food intake of sibutramine or orlistat (Figure 2B).

Table 2 Effect of empagliflozin and sibutramine on the plasma levels of various metabolic parameters on day 28 in diet-induced obese rats

\begin{tabular}{|c|c|c|c|c|}
\hline & Glucose $(\mathrm{mM})$ & Insulin $(\mathrm{ng} / \mathrm{mL})$ & Leptin (ng/mL) & $\mathrm{HbA}_{\mathrm{lc}}(\%)$ \\
\hline Baseline & $7.27 \pm 0.11$ & $3.87 \pm 0.25$ & $34.2 \pm 1.7$ & $6.9 \pm 0.03$ \\
\hline Vehicle & $7.37 \pm 0.30$ & $3.42 \pm 0.49$ & $26.6 \pm 1.8$ & $6.85 \pm 0.04$ \\
\hline Empagliflozin (10 mg/kg PO) & $6.70 \pm 0.11$ & $2.47 \pm 0.22$ & $22.5 \pm 2.3$ & $6.87 \pm 0.07$ \\
\hline Empagliflozin (30 mg/kg PO) & $6.60 \pm 0.17$ & $2.16 \pm 0.35$ & $17.8 \pm 2.2 * *$ & $6.83 \pm 0.06$ \\
\hline Empagliflozin (60 mg/kg PO) & $6.73 \pm 0.21^{*}$ & $2.49 \pm 0.26$ & $14.6 \pm 1.5^{* * *}$ & $6.88 \pm 0.05$ \\
\hline \multirow[t]{2}{*}{ Sibutramine (5 mg/kg PO) } & $5.77 \pm 0.33 * * *$ & $1.83 \pm 0.25 * *$ & $17.9 \pm 1.4^{* *}$ & $6.8 I \pm 0.06$ \\
\hline & Adiponectin $(\mu \mathrm{g} / \mathrm{mL})$ & TAG (mM) & NEFA $(\mu \mathrm{M})$ & HOMA-IR \\
\hline Baseline & $25.3 \pm 0.8$ & $0.310 \pm 0.02$ & $1,034 \pm 27$ & $27.30 \pm 1.91$ \\
\hline Vehicle & $23.2 \pm 1.1$ & $0.292 \pm 0.03$ & $982 \pm 72$ & $24.74 \pm 4.01$ \\
\hline Empagliflozin (10 mg/kg PO) & $20.5 \pm 0.8$ & $0.27 I \pm 0.02$ & $\mathrm{I}, 124 \pm 80$ & $16.16 \pm 1.50 *$ \\
\hline Empagliflozin (30 mg/kg PO) & $21.2 \pm 0.9$ & $0.32 I \pm 0.02$ & $\mathrm{I}, \mathrm{I} 44 \pm 73$ & $13.72 \pm 2.24 *$ \\
\hline Empagliflozin (60 mg/kg PO) & $19.4 \pm 0.8^{*}$ & $0.368 \pm 0.04$ & $|, 086 \pm 4|$ & $16.37 \pm 1.96 *$ \\
\hline Sibutramine (5 mg/kg PO) & $18.4 \pm 1.3 *$ & $0.179 \pm 0.03 * *$ & $1,164 \pm 115$ & $10.07 \pm 1.73 * * *$ \\
\hline
\end{tabular}

Notes: $* P<0.05,{ }^{*} P<0.01, * * * P<0.001$ (significant differences from the vehicle-treated group). Samples were taken after a 4-hour fast. Data are means and standard error $(n=6-10)$. Baseline values are those determined shortly prior to the onset of drug treatment.

Abbreviations: PO, per os (by mouth); TAG, triacylglycerol; NEFA, nonesterified fatty acid; HOMA-IR, homeostasis-model assessment of insulin resistance; HbA ${ }_{\text {Ic }}$ glycated hemoglobin. 


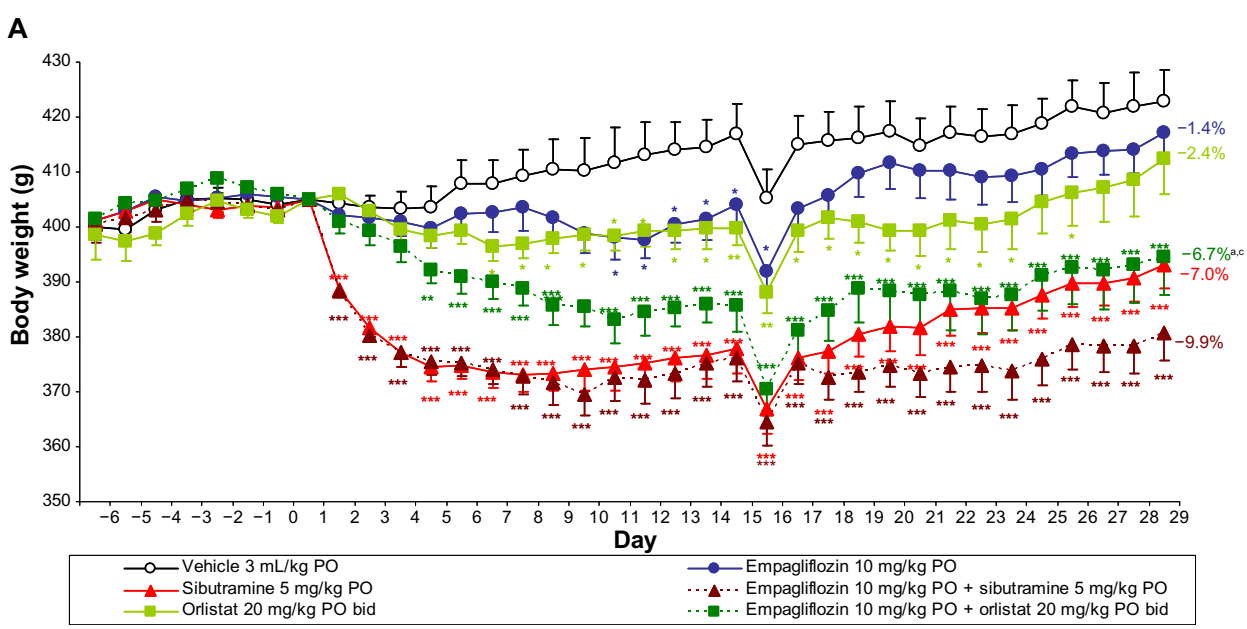

B

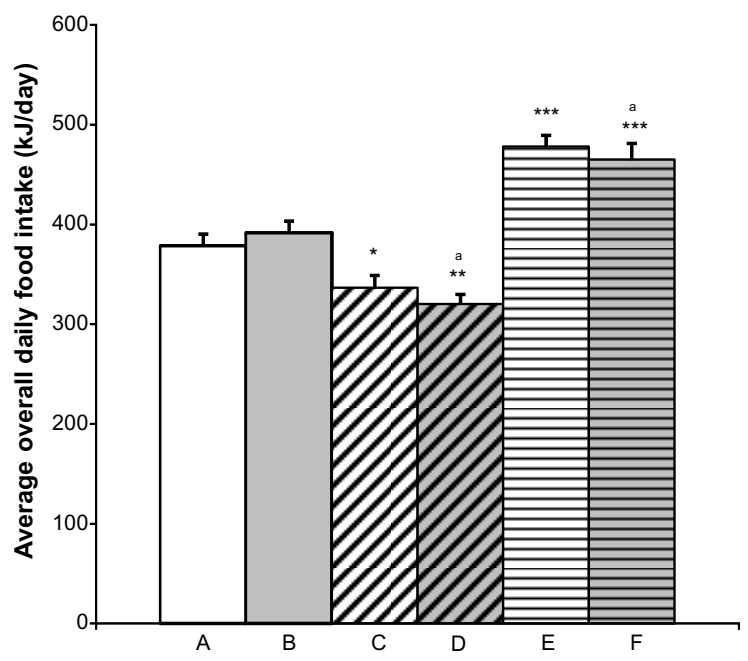

$\square$ A: Vehicle PO

$\square$ B: Empagliflozin $10 \mathrm{mg} / \mathrm{kg}$ PO

$\square$ C: Sibutramine $5 \mathrm{mg} / \mathrm{kg}$ PO

口: Empagliflozin $10 \mathrm{mg} / \mathrm{kg}$ PO + sibutramine $5 \mathrm{mg} / \mathrm{kg}$ PO

口E: Orlistat $20 \mathrm{mg} / \mathrm{kg}$ PO bid

曰F: Empagliflozin $10 \mathrm{mg} / \mathrm{kg}$ PO + orlistat $20 \mathrm{mg} / \mathrm{kg}$ PO bid
C

Plasma glucose
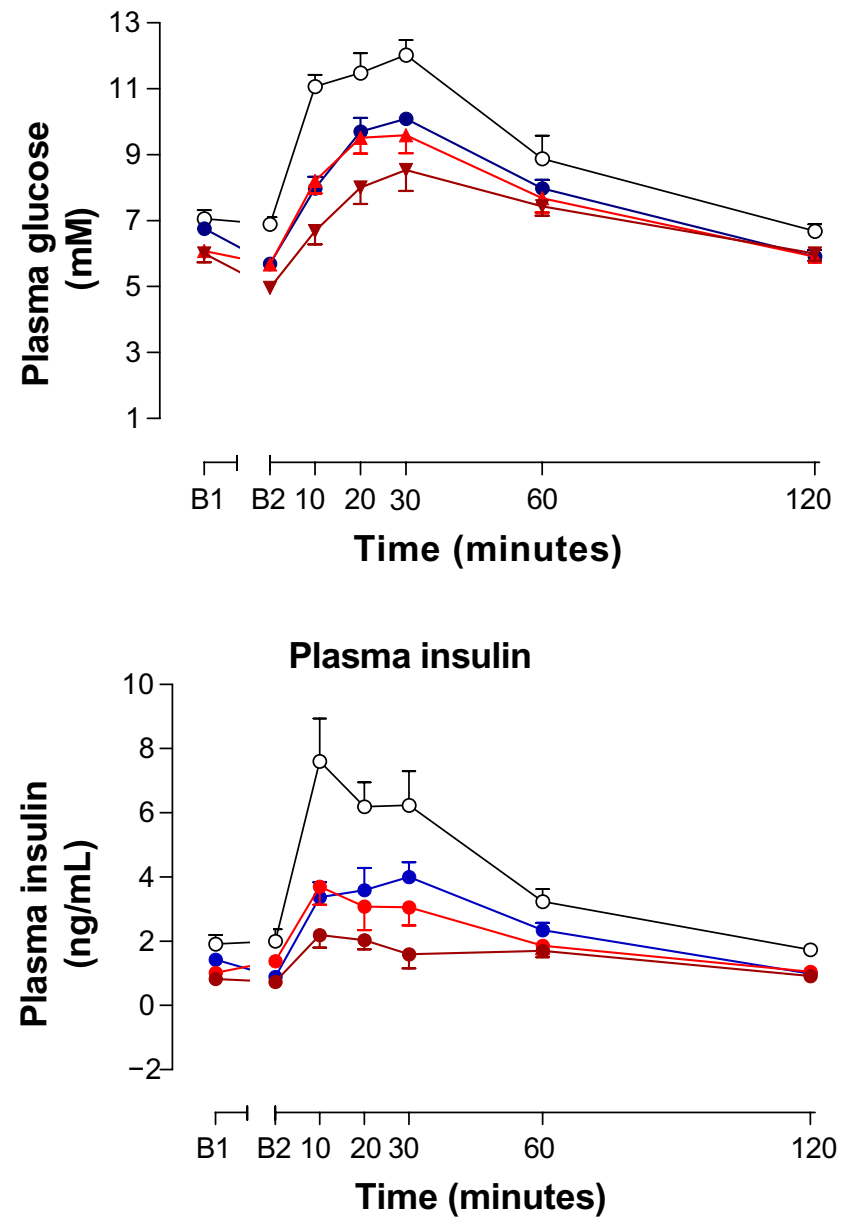

-o- Vehicle $3 \mathrm{~mL} / \mathrm{kg}$ po

$\longrightarrow$ Empagliflozin $10 \mathrm{mg} / \mathrm{kg}$ po

$\leftarrow$ Sibutramine $5 \mathrm{mg} / \mathrm{kg}$ po

$\rightarrow \quad$ Empagliflozin $10 \mathrm{mg} / \mathrm{kg}$ po + sibutramine $5 \mathrm{mg} / \mathrm{kg}$ po

Figure 2 (Continued) 
D

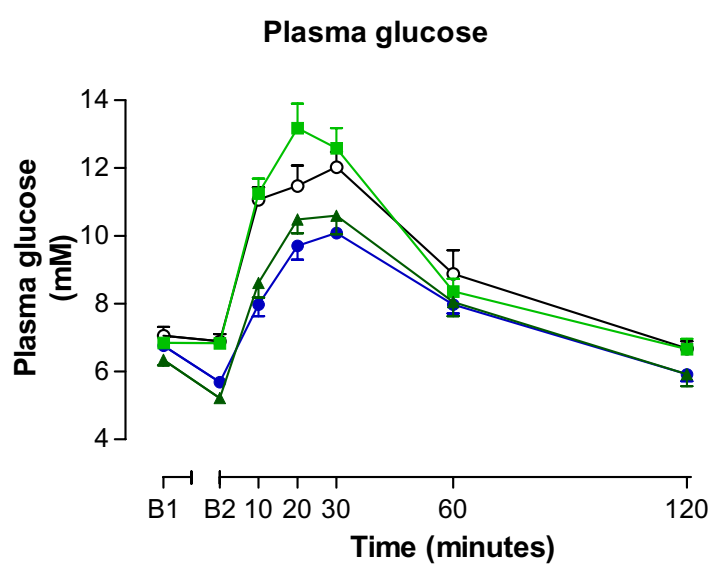

Plasma insulin

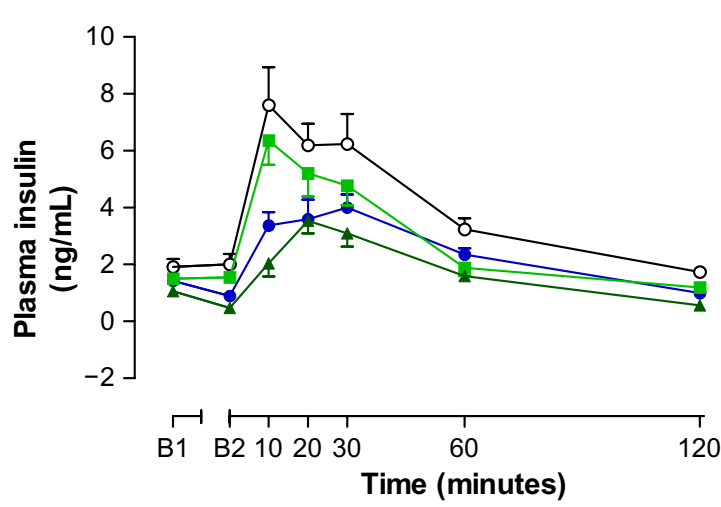

-o- Vehicle $3 \mathrm{~mL} / \mathrm{kg}$ po

$\rightarrow$ Empagliflozin $10 \mathrm{mg} / \mathrm{kg}$ po

$\rightarrow$ Orlistat $20 \mathrm{mg} / \mathrm{kg}$ bid

$\leftarrow$ Empagliflozin $10 \mathrm{mg} / \mathrm{kg}$ po

+ orlistat $20 \mathrm{mg} / \mathrm{kg}$ po bid
E
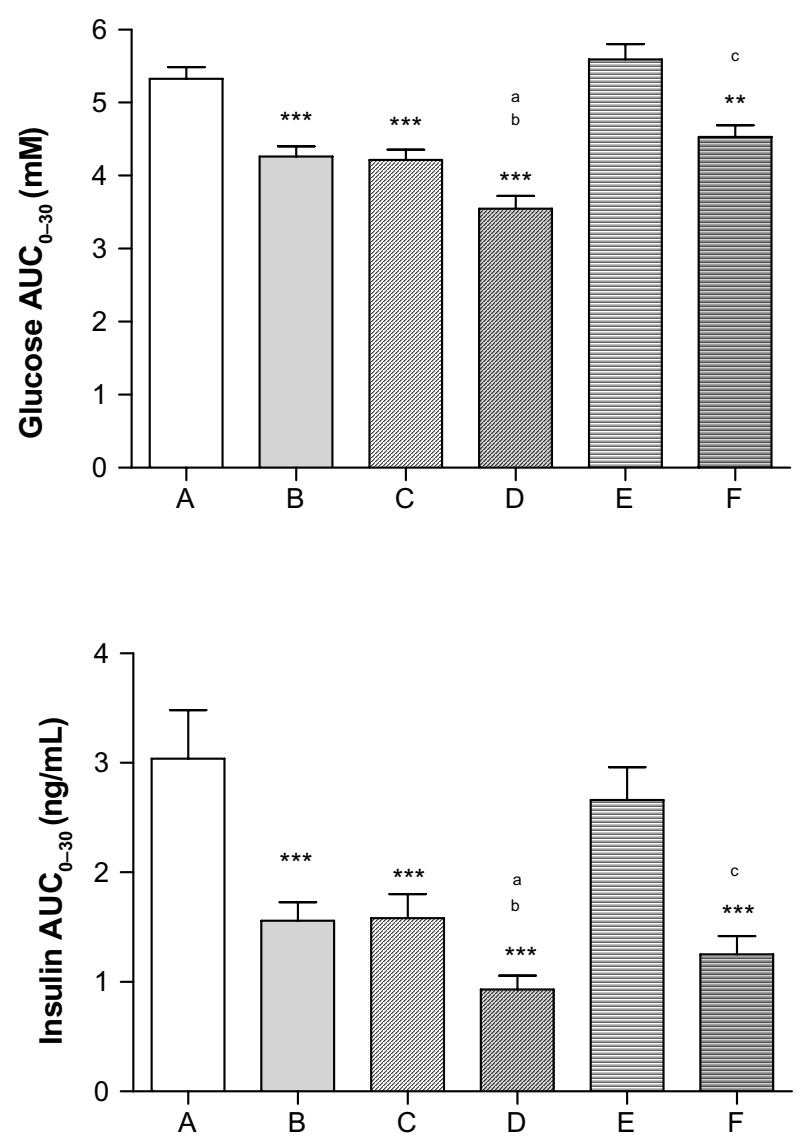

Figure 2 The effect of empagliflozin, sibutramine, and orlistat both alone and in combination on rat body weight (A), food intake (B) and day 30 glucose tolerance $(\mathbf{C}-\mathbf{E})$. Notes: $* P<0.05$; $* * P<0.01$; $* * * P<0.00 I$; ${ }^{a}$ significant difference from the empagliflozin group $(P<0.01)$; ${ }^{b}$ significant difference from the sibutramine group $(P<0.05)$; ${ }^{c}$ significant difference from the orlistat group $(P<0.05)$ Data are means and standard error $(n=8-10)$. For clarity, the glucose and insulin excursions are separated for the empagliflozin/ sibutramine and empagliflozin/orlistat combinations (C and D). In addition, for clarity, significant differences from control and between individual groups are not illustrated in $\mathbf{C}$ and $\mathbf{D}$ (for differences, see $\mathbf{E}$ ).

Abbreviations: PO, per os (by mouth); bid, bis in die (twice daily); AUC, area under the curve.

In contrast to empagliflozin $(P<0.05)$ and sibutramine $(P<0.01)$, which both increased average daily water intake over the study duration, orlistat did not significantly affect water consumption $(P=0.07)$. The combination of empagliflozin with either sibutramine $(P<0.01)$ or orlistat $(P<0.001)$ led to significantly increased average daily water intake over the study duration compared to vehicle-treated controls (for clarity, data not shown).

Sibutramine and orlistat significantly and selectively reduced carcass fat when administered alone compared to both vehicle-treated controls $(P<0.05$, Table 3$)$. When dosed in combination, the reduction in body fat $(P<0.001)$ induced by empagliflozin and sibutramine was significantly greater than that observed with either drug alone $(P<0.05$, Table 3). The combination of orlistat and empagliflozin significantly reduced body fat $(P<0.01)$ compared to controls. However, although the combination effect was greater than that with empagliflozin alone $(P<0.01$, Table 3$)$ it was not significantly different to that observed with orlistat alone $(P=0.55$, Table 3$)$. 
Table 3 The effect of empagliflozin, sibutramine, and orlistat both alone and in combination with empagliflozin on body composition of diet-induced obese rats at study conclusion

\begin{tabular}{|c|c|c|c|}
\hline & Water (g) & Fat (g) & Protein $(\mathrm{g})$ \\
\hline Vehicle & $211.0 \pm 2.1$ & $116.4 \pm 7.1$ & $68.2 \pm 0.9$ \\
\hline Empagliflozin (10 mg/kg PO) & $209.0 \pm 3.8$ & $1 \mid 3.8 \pm 2.7$ & $66.4 \pm 1.0$ \\
\hline Sibutramine (5 mg/kg PO) & $204.8 \pm 3.4$ & $95.5 \pm 4.6 *$ & $67.1 \pm 1.2$ \\
\hline $\begin{array}{l}\text { Empagliflozin ( } 10 \mathrm{mg} / \mathrm{kg} \mathrm{PO}) / \\
\text { sibutramine ( } 5 \mathrm{mg} / \mathrm{kg} \mathrm{PO})\end{array}$ & $207.6 \pm 2.8$ & $75.2 \pm 6.0 * * *, a, b$ & $68.1 \pm 0.9$ \\
\hline Orlistat (20 mg/kg PO BID) & $216.3 \pm 3.5$ & $93.7 \pm 4.4^{*}$ & $69.9 \pm 1.4$ \\
\hline $\begin{array}{l}\text { Empagliflozin (I0 mg/kg PO)/ } \\
\text { orlistat (20 mg/kg PO BID) }\end{array}$ & $206.9 \pm 2.1$ & $88.4 \pm 7.8^{* *, a}$ & $66.9 \pm 0.8$ \\
\hline
\end{tabular}

Notes: $* P<0.05, * * P<0.01$, $* * * P<0.001$ (significant differences from the vehicletreated group); $P<0.01$ (significant differences from the empagliflozin group); ${ }^{b} P<0.05$ (significant difference from the sibutramine group). Data are means and standard error $(n=9-10)$.

Abbreviations: PO, per os (by mouth); BID, bis in die (twice daily).

Empagliflozin $(P<0.001)$ and sibutramine $(P<0.001)$ significantly improved glucose tolerance (eg, reduced glucose $\mathrm{AUC}_{0-30}$ ) when administered alone (Figure 2C and $\mathrm{E}$ ). These improvements were associated with significant reductions in plasma insulin compared to vehicle-treated controls $\left(\mathrm{AUC}_{0-30}, P<0.001\right.$; Figure $2 \mathrm{C}$ and $\left.\mathrm{E}\right)$. When combined with empagliflozin, the effect of sibutramine in reducing both plasma glucose and insulin was increased compared to that of the individual drug treatments (Figure 2C), leading to a statistically significant reduction in $\mathrm{AUC}_{0-30}(P<0.05$, Figure 2E). Orlistat did not significantly affect glucose tolerance (Figure 2D) when dosed alone, although a significant reduction in both the plasma glucose $(P<0.01)$ and insulin $\mathrm{AUC}_{0-30}(P<0.001)$ was observed when orlistat was administered in combination with empagliflozin compared to vehicle-treated controls (Figure 2E).

Blood samples were taken after an overnight fast on both days 16 and 30 . There were no substantial differences between the time points. Accordingly, for clarity, only the day 30 data are presented (Table 4). Both empagliflozin and sibutramine had significantly reduced plasma insulin by day 30 when administered alone $(P<0.05$ and $P<0.001$, respectively; Table 4 ) though only sibutramine significantly reduced plasma glucose $(P<0.001)$ and HOMA-IR. The combination of empagliflozin with sibutramine significantly reduced plasma glucose $(P<0.001)$, insulin $(P<0.001)$, TAG $(P<0.05)$, and leptin $(P<0.001)$, and further improved insulin sensitivity (decrease in HOMA-IR, $P<0.001$ ) compared to vehicle-treated controls at day 30 (Table 4 ). By day 30 , the combination of empagliflozin and orlistat had significantly reduced plasma glucose $(P<0.01)$ and insulin $(P<0.001)$, while orlistat alone had not. Neither orlistat nor empagliflozin improved insulin resistance (HOMA-IR) in the combination experiment when given alone. However, in contrast, the combination of orlistat with empagliflozin induced a marked and significant improvement in insulin sensitivity (decreased HOMA-IR, $P<0.001)$. Both sibutramine $(P<0.05)$ and orlistat $(P<0.05)$ reduced plasma leptin, and in both cases this reduction was increased in the presence of empagliflozin $(P<0.05$ versus orlistat, Table 4$)$. However, this difference did not quite achieve statistical significance in the case of sibutramine $(P=0.16)$.

\section{Discussion}

The present study demonstrates that empagliflozin, a selective SGLT2 inhibitor under development for type 2 diabetes, reduces body weight in addition to improving glucose control in a preclinical model of DIO with insulin resistance. ${ }^{13-15}$ Female rats were used in the present study, since obesity is more prevalent in women than in men. ${ }^{1}$ The reduction in plasma leptin was consistent with the empagliflozin-induced weight loss, and body-composition analysis confirmed that the

Table 4 The effect of empagliflozin, sibutramine, and orlistat both alone and in combination with empagliflozin on the plasma levels of various metabolic parameters on day 30 in diet-induced obese rats

\begin{tabular}{|c|c|c|c|c|c|}
\hline & Glucose (mM) & Insulin (ng/mL) & HOMA-IR & Leptin $(\mathrm{ng} / \mathrm{mL})$ & TAG (mM) \\
\hline Baseline & $5.91 \pm 0.07$ & $1.98 \pm 0.12$ & $11.36 \pm 0.79$ & $9.2 \pm 0.8$ & $0.219 \pm 0.01$ \\
\hline Vehicle & $7.09 \pm 0.30$ & $2.04 \pm 0.33$ & $13.94 \pm 2.65$ & $\mid 4.1 \pm 1.5$ & $0.263 \pm 0.05$ \\
\hline Empagliflozin (10 mg/kg PO) & $6.75 \pm 0.15$ & $1.34 \pm 0.15^{*}$ & $8.88 \pm 1.10$ & $12.2 \pm 1.3$ & $0.193 \pm 0.03$ \\
\hline Sibutramine (5 mg/kg PO) & $6.08 \pm 0.17^{* * *}$ & $0.95 \pm 0.12^{* * *}$ & $5.67 \pm 0.73 * * *$ & $10.2 \pm 1.3^{*}$ & $0.186 \pm 0.03$ \\
\hline $\begin{array}{l}\text { Empagliflozin ( } 10 \mathrm{mg} / \mathrm{kg} \mathrm{PO}) / \\
\text { sibutramine ( } 5 \mathrm{mg} / \mathrm{kg} \mathrm{PO})\end{array}$ & $5.73 \pm 0.3 \mid * * *, a$ & $0.82 \pm 0.12^{* * *, a}$ & $4.67 \pm 0.72^{* * *, a}$ & $7.8 \pm 1.1 * * *, a$ & $0.166 \pm 0.04^{*}$ \\
\hline Orlistat (20 mg/kg PO BID) & $6.77 \pm 0.19$ & $1.47 \pm 0.19$ & $9.89 \pm 1.28$ & $10.3 \pm 0.8 *$ & $0.323 \pm 0.04$ \\
\hline $\begin{array}{l}\text { Empagliflozin ( } 10 \mathrm{mg} / \mathrm{kg} \text { PO)/orlistat } \\
(20 \mathrm{mg} / \mathrm{kg} \text { PO BID) }\end{array}$ & $6.27 \pm 0.17^{* *}$ & $0.94 \pm 0.15^{* * *, c}$ & $5.65 \pm 1.05^{* * *, c}$ & $6.3 \pm 0.9 * * *, a, c$ & $0.27 I \pm 0.04$ \\
\hline
\end{tabular}

Notes: $* P<0.05, * * P<0.01$, $* * * P<0.001$ (significant differences from the vehicle-treated group); $P<0.05$ (significant differences from the empagliflozin group); $P<0.05$ (significant differences from the orlistat group). Samples were taken after an overnight fast. Data are means and standard error ( $\mathrm{n}=9-10)$. Baseline values are those determined shortly prior to the onset of drug treatment.

Abbreviations: TAG, triacylglycerol; HOMA-IR, homeostasis-model assessment of insulin resistance; PO, per os (by mouth); BID, bis in die (twice daily). 
reduction in body weight was attributable to a selective reduction in body fat, since body water and protein were not significantly altered by drug treatment. Hence, drug-induced cachexia is an unlikely explanation of the weight loss. Consistent with the known activity of the drug in diabetic animals, ${ }^{12}$ empagliflozin treatment was associated with an increase in UGE. The calorie loss associated with this increased UGE is likely to be a major cause of the weight loss observed in the obese animals, since the significant effect of empagliflozin in decreasing body weight was not necessarily associated with reduced food intake. Indeed, empagliflozin only significantly reduced food intake compared to vehicle-treated controls at the highest dose tested. Reasons for this hypophagia may include a hitherto-unknown action of the compound to promote satiation, or alternatively be attributed to the elevated water intake of the animals, since there are at least some data suggesting that increased water consumption with meals has been found to reduce energy intake and therefore increase weight loss in obese patients. ${ }^{19}$ Interestingly, even at lower doses of empagliflozin, animals did not appear to compensate for the loss of calories from urine by increasing food intake. In the present study, it was not possible to demonstrate that the urinary calorie loss accounted for the body-weight loss, since urine was collected over only a 7-hour period and in the absence of food. An energy-balance study measuring daily body weight and food and water intake, in addition to collecting all urine and feces, would be required for this analysis.

Although, sibutramine was withdrawn from the market in 2010 due to the increased risk of serious, nonfatal cardiovascular events, such as stroke or myocardial infarction, ${ }^{20}$ the compound was used as a reference agent due to the extensive preclinical and clinical literature available. In agreement with the reported activity of the compound in preclinical models ${ }^{13-15,21}$ and the clinic, ${ }^{22,23}$ sibutramine not only reduced body weight but also selectively reduced body fat in the present study. Although the lipase inhibitor orlistat reduces body weight in the clinic ${ }^{23,24}$ and has previously reduced body weight in the DIO rat at this dose, ${ }^{25}$ the effect of the drug in reducing body weight in the present study was modest, such that a statistically significant reduction from vehicle-treated controls was not evident at study termination. Furthermore, in contrast to clinical data, ${ }^{26}$ in the present rat study orlistat treatment was associated with a robust hyperphagia, presumably to compensate for the action of the drug to prevent the intestinal absorption of fat.

The degree of weight loss evident in the study appeared to be related to the starting body weight of the animals. Specifically, both empagliflozin and sibutramine had a greater effect in reducing body weight in the initial study, where the animals exhibited increased adiposity and a greater degree of insulin resistance. The reason for this is likely that animals with greater adiposity offer a larger window for successful and marked drug intervention. If such a finding extends to the clinic, then this may confer an added advantage of empagliflozin in the treatment of obese patients with type 2 diabetes compared to antidiabetic treatments that may induce weight gain. ${ }^{27}$ The lower body weight of the second study cohort may also be one reason the effect of orlistat to reduce body weight was not marked. The weight loss induced by sibutramine and empagliflozin (60 mg/kg PO) was associated with a statistically significant reduction in plasma adiponectin compared to vehicle-treated controls. The reason for this is unclear, since body-weight loss is typically associated with increased levels of this adipokine. ${ }^{28}$ Although the present results were unexpected, there is at least one report in rats that adiponectin levels are associated with overweight and insulin resistance, although these animals were also hypertensive. ${ }^{29}$

Consistent with the reported effect of the drug in diabetic animals ${ }^{12}$ and SGLT2 inhibitors in patients with type 2 diabetes, ${ }^{7,8}$ empagliflozin dose-dependently reduced plasma glucose, with a statistically significant effect only at the highest dose tested. However, the moderate effects observed in this particular model are likely to be attributable to the cafeteria diet-fed rats exhibiting plasma glucose and blood $\mathrm{HbA}_{1 \mathrm{c}}$ levels within a normal range. Although the cafeteria-fed animals were not diabetic, they exhibited a moderate hyperinsulinemia associated with insulin resistance. Accordingly, the effect of sibutramine in reducing body weight in the model was associated with an improvement in insulin sensitivity and a corresponding reduction in plasma insulin and HOMA-IR. As expected for an antidiabetic drug, empagliflozin also significantly improved insulin sensitivity (either HOMA-IR or plasma-insulin levels) although reductions in body weight were modest in comparison to sibutramine. Furthermore, consistent with other SGLT2 inhibitors ${ }^{30}$ empagliflozin, and the SNRI sibutramine, but not orlistat, improved glucose tolerance (without stimulating insulin secretion), as illustrated by significantly reducing the glucose and insulin AUC in an oral glucose-tolerance test. The improvements in glucose tolerance and plasma insulin in the case of the antiobesity agent sibutramine are consistent with clinical findings,${ }^{31}$ though are likely to be secondary to the weight loss induced by the compound.

Interestingly, the combination treatment of empagliflozin with either sibutramine or orlistat led to increased body weight or body fat loss compared to that observed with either 
drug alone. Indeed, the body-weight reduction observed with empagliflozin in combination with sibutramine or orlistat was greater than the additive effects of the drugs when dosed alone, raising the possibility of a synergistic interaction of the combination. However, this apparently synergistic relationship may simply be a consequence of the smaller effect of the $10 \mathrm{mg} / \mathrm{kg}$ dose of empagliflozin alone in this combination study compared to the initial dose-response experiment. Indeed, an isobologram approach with more detailed doseresponse curves of the drugs alone and in combination would be required to directly address this hypothesis. In the case of the combination treatments tested in the present study, over $90 \%$ of the weight loss was attributable to fat loss. When coupled with the antidiabetic effect of empagliflozin, the combination further improved the glucose-control and plasma parameters (insulin, HOMA-IR, leptin, TAG) of animals treated with orlistat or sibutramine alone.

The present data suggest a number of potential advantages in using an SGLT2 inhibitor as part of a dual-therapy approach in the treatment of type 2 diabetes or potentially obesity. Hence, in the case of type 2 diabetes, dual therapy of an SGLT2 inhibitor with such a drug as sibutramine or orlistat may have the advantage of directly controlling hyperglycemia, in addition to increasing the action of the other therapy to reduce body weight, with the commensurate benefits on insulin sensitivity and glucose control subsequently manifest. Sibutramine was withdrawn from sale in Europe by European Medicines Agency (2010) and from the US voluntarily by Abbott Laboratories. Hence, the combination of an SGLT2 inhibitor would need to be evaluated clinically with currently approved weight-loss drugs, such as the 5- $\mathrm{HT}_{2 \mathrm{C}}$-receptor agonist lorcaserin (Belviq ${ }^{\circledR}$; Arena Pharmaceuticals, San Diego, CA, USA), the lipase inhibitor orlistat (Xenical ${ }^{\circledR}$; Roche, Basel, Switzerland), or the combination of phentermine and topiramate Qsymia ${ }^{\circledR}$ (VIVUS, Inc., Mountain View, CA, USA). Interestingly, there is evidence that sibutramine reduces food intake and presumably body weight, at least in part, through activation of $5-\mathrm{HT}_{2 \mathrm{C}}$ receptors. ${ }^{32}$

In the case of obese patients, the combination of an antiobesity drug with empagliflozin may not only further control the body weight of the patients, but more interestingly may slow down or prevent the progression from obesity to diabetes. In contrast to other drug $\operatorname{classes}^{27}$ (eg, thiazolidinediones, sulfonylureas, insulin, etc), the use of SGLT2 inhibitors is associated with weight loss, predominantly through reduction of total fat mass, ${ }^{7,33}$ rather than weightgain induction, ${ }^{27}$ which is a major causative factor in the development of diabetes. In addition, the insulin-independent action of SGLT2 inhibitors and the associated reduced risk of hypoglycaemia ${ }^{9,10}$ is an important characteristic, especially if such drugs were also to be considered for use in patient populations, such as those in whom moderate hyperglycemia or euglycemia may be evident.

The major reported side effects associated with the use of such SGLT2 inhibitors as empagliflozin are infection of the urinary and genital tracts. ${ }^{34}$ Accordingly, if SGLT2 inhibitors were to be used in combination with treatments that reduce body weight in prediabetic or type 2 diabetic patients, then it is likely that these adverse effects will also be manifest. That said, typically the incidence is low $\left(3 \%-13 \%\right.$ of patients $\left.^{34}\right)$, and SGLT2 inhibitors are regarded as safe and effective treatments for hyperglycemia in type 2 diabetes. ${ }^{35}$

In conclusion, the present data show that chronic administration of the novel SGLT2 inhibitor empagliflozin reduces body weight, body fat, plasma leptin, and insulin resistance, and improves glucose tolerance in the absence of insulin stimulation in obese rats. In addition, the combination of empagliflozin with clinically effective treatments for obesity demonstrates significantly improved outcomes in body-weight or body-fat reduction, plasma leptin, and glucose control, which in general are likely to be additive in nature. In light of clinical data detailing the benefits of weight-loss agents on glycemic control and progression to diabetes, ${ }^{31}$ such combination studies may elucidate improved strategies for the treatment of type 2 diabetes or obesity in clinical practice.

\section{Author contributions}

SCC, RG, MM, TK, EM and SPV were responsible for the study's conception, design, and interpretation of data. SPV, SCC, RG, EM, and MM drafted the article, and the article was revised and the final version approved by all authors. $\mathrm{KRH}$ and KD were responsible for the plasma- and bodycomposition analysis and data interpretation.

\section{Disclosure}

RG, EM, MM, and TK are employees of Boehringer Ingelheim Pharma GmbH and Co. SPV, KD, KRH, and SCC are employees of RenaSci Ltd, a fee-for-service contract research organization.

\section{References}

1. World Health Organization. Obesity and overweight. Available from: http://www.who.int/mediacentre/factsheets/fs311/en/index.html. Accessed March 15, 2014.

2. Cecchini M, Sassi F, Lauer JA, Lee YY, Guajardo-Barron V, Chisholm D. Tackling of unhealthy diets, physical inactivity, and obesity: health effects and cost-effectiveness. Lancet. 2010;376(9754):1775-1784. 
3. Heal DJ, Gosden J, Smith SL. Regulatory challenges for new drugs to treat obesity and comorbid metabolic disorders. Br J Clin Pharmacol. 2009;68(6):861-874.

4. Jindal A, Brietzke S, Sowers JR. Obesity and the cardiorenal metabolic syndrome: therapeutic modalities and their efficacy in improving cardiovascular and renal risk factors. Cardiorenal Med. 2012;2(4): 314-327.

5. Phung OJ, Scholle JM, Talwar M, et al. Effect of noninsulin antidiabetic drugs added to metformin therapy on glycemic control, weight gain, and hypoglycemia in type 2 diabetes. JAMA. 2010;303(14):1410-1418.

6. Mather A, Pollock C. Glucose handling by the kidney. Kidney Int Suppl. 2011;(120):S1-S6.

7. Zhang L, Feng Y, List J, Kasichayanula S, Pfister M. Dapagliflozin treatment in patients with different stages of type 2 diabetes mellitus: effects on glycaemic control and body weight. Diabetes Obes Metab. 2010;12(6):510-516.

8. List JF, Woo V, Morales E, Tang W, Fiedorek FT. Sodium-glucose cotransport inhibition with dapagliflozin in type 2 diabetes. Diabetes Care. 2009;32(4):650-657.

9. Bailey CJ. SGLT2 inhibitors: glucuretic treatment for type 2 diabetes. Br J Diabetes Vasc Dis. 2010;10(4):193-199.

10. Bailey CJ. Renal glucose reabsorption inhibitors to treat diabetes. Trends Pharmacol Sci. 2011;32(2):63-71.

11. Grempler R, Thomas L, Eckhardt M, et al. Empagliflozin, a novel selective sodium glucose cotransporter-2 (SGLT-2) inhibitor: characterisation and comparison with other SGLT-2 inhibitors. Diabetes Obes Metab. 2012;14(1):83-90.

12. Thomas L, Grempler R, Eckhardt M, et al. Long-term treatment with empagliflozin, a novel, potent and selective SGLT-2 inhibitor, improves glycaemic control and features of metabolic syndrome in diabetic rats. Diabetes Obes Metab. 2012;14(1):94-96.

13. Dickinson K, North TJ, Anthony DM, Jones RB, Heal DJ. Evaluation of a simplified cafeteria model for the induction of insulin resistant obesity in rats. Int J Obes. 1998;22 Suppl 3: S19.

14. Fisas A, Codony X, Romero G, et al. Chronic 5-HT6 receptor modulation by E-6837 induces hypophagia and sustained weight loss in dietinduced obese rats. Br J Pharmacol. 2006;148(7):973-983.

15. Vickers SP, Jackson HC, Cheetham SC. The utility of animal models to evaluate novel anti-obesity agents. Br J Pharmacol. 2011;164(4): $1248-1262$.

16. Häring HU, Merker L, Seewaldt-Becker E. Empagliflozin as add-on to metformin plus sulfonylurea in patients with type 2 diabetes: a 24 -week, randomized, double-blind, placebo-controlled trial. Diabetes Care 2013;36(11):3396-3404.

17. Kovacs CS, Seshiah V, Swallow R. Empagliflozin improves glycaemic and weight control as add-on therapy to pioglitazone or pioglitazone plus metformin in patients with type 2 diabetes: a 24-week, randomized, placebo-controlled trial. Diabetes Obes Metab. Epub August 1, 2013.

18. Dickinson K, Mody N, Slater N, et al. The effect of chronic administration of sibutramine on body composition in dietary-induced, obese female Wistar rats - an evaluation of the Foss FoodScan ${ }^{\mathrm{TM}}$ analyser. 2008. Available from: http://pa2online.org/abstract/abstract. jsp?abid=29118. Accessed March 15, 2014.

19. Dennis EA, Dengo AL, Comber DL, et al. Water consumption increases weight loss during a hypocaloric diet intervention in middle-aged and older adults. Obesity (Silver Spring). 2010;18(2):300-307.
20. James WP, Caterson ID, Coutinho W. Effect of sibutramine on cardiovascular outcomes in overweight and obese subjects. $N$ Engl $\mathrm{J}$ Med. 2010;363(10):905-917.

21. Hansen HH, Hansen G, Tang-Christensen M, et al. The novel triple monoamine reuptake inhibitor tesofensine induces sustained weight loss and improves glycemic control in the diet-induced obese rat: comparison to sibutramine and rimonabant. Eur J Pharmacol. 2010;636(1-3):88-95.

22. Bray GA, Ryan DH, Gordon D, Heidingsfelder S, Cerise F, Wilson K A double-blind randomized placebo-controlled trial of sibutramine. Obes Res. 1996;4(3):263-270.

23. Gokcel A, Gumurdulu Y, Karakose H, et al. Evaluation of the safety and efficacy of sibutramine, orlistat and metformin in the treatment of obesity. Diabetes Obes Metab. 2002;4(1):49-55.

24. Sjöström L, Rissanen A, Andersen T. Randomised placebo-controlled trial of orlistat for weight loss and prevention of weight regain in obese patients. Lancet. 1998;352(9123):167-172.

25. Jackson HC, Dickinson K, Jones RB, Schumacher C, Jensen C. Comparison of the effects of sibutramine and orlistat on body weight, food and water intake and body composition in dietary-induced obese rats. Poster presented at: Society for Neuroscience Meeting; October 23-27, 2004; San Diego, CA.

26. Svendsen M, Helgeland M, Tonstad S. The long-term influence of orlistat on dietary intake in obese subjects with components of metabolic syndrome. J Hum Nutr Diet. 2009;22(1):55-63.

27. Fonseca V. Effect of thiazolidinediones on body weight in patients with diabetes mellitus. Am J Med. 2003;115 Suppl 8A:42S-48S.

28. Dridi S, Taouis M. Adiponectin and energy homeostasis: consensus and controversy. J Nutr Biochem. 2009;20(11):831-839.

29. Rodríguez A, Catalán V, Becerril S, et al. Impaired adiponectin-AMPK signalling in insulin-sensitive tissues of hypertensive rats. Life Sci. 2008;83(15-16):540-549.

30. Fujimori Y, Katsuno K, Nakashima I, Ishikawa-Takemura Y, Fujikura H, Isaji M. Remogliflozin etabonate, in a novel category of selective low-affinity sodium glucose cotransporter (SGLT2) inhibitors, exhibits antidiabetic efficacy in rodent models. $J$ Pharmacol Exp Ther. 2008;327(1):268-276.

31. Lloret-Linares C, Greenfield JR, Czernichow S. Effect of weightreducing agents on glycaemic parameters and progression to type 2 diabetes: a review. Diabet Med. 2008;25(10):1142-1150.

32. Jackson HC, Bearham MC, Hutchins LJ, Mazurkiewicz SE, Needham AM, Heal DJ. Investigation of the mechanisms underlying the hypophagic effects of the 5-HT and noradrenaline reuptake inhibitor, sibutramine, in the rat. Br J Pharmacol. 1997;121(8):1613-1618.

33. Bolinder J, Ljunggren Ö, Kullberg J, et al. Effects of dapagliflozin on body weight, total fat mass, and regional adipose tissue distribution in patients with type 2 diabetes mellitus with inadequate glycemic control on metformin. Clin Endocrinol Metab. 2012;97(3):1020-1031.

34. Ferrannini E, Berk A, Hantel S, et al. Long-term safety and efficacy of empagliflozin, sitagliptin, and metformin: an active-controlled, parallelgroup, randomized, 78-week open-label extension study in patients with type 2 diabetes. Diabetes Care. 2013;36(12):4015-4021.

35. Musso G, Gambino R, Cassader M, Pagano G. A novel approach to control hyperglycemia in type 2 diabetes: sodium glucose co-transport (SGLT) inhibitors: systematic review and meta-analysis of randomized trials. Ann Med. 2012;44(4):375-393.

Diabetes, Metabolic Syndrome and Obesity: Targets and Therapy

\section{Publish your work in this journal}

Diabetes, Metabolic Syndrome and Obesity: Targets and Therapy is an international, peer-reviewed open-access journal committed to the rapid publication of the latest laboratory and clinical findings in the fields of diabetes, metabolic syndrome and obesity research Original research, review, case reports, hypothesis formation, expert opinion and commentaries are all considered for publication. The manuscript management system is completely online and includes a very quick and fair peer-review system, which is all easy to use. Visit http://www.dovepress.com/testimonials.php to read real quotes from published authors. 